\title{
Evaluation of image quality at the detector's edge of dedicated breast positron emission tomography
}

\author{
Yoko Satoh ${ }^{1,2^{*}}$ D, Utaroh Motosugi ${ }^{3}$, Masamichi Imai ${ }^{1}$, Yoshie Omiya ${ }^{2}$ and Hiroshi Onishi ${ }^{2}$
}

\author{
* Correspondence: ysatoh@ypic.jp \\ ${ }^{1}$ Yamanashi PET Imaging Clinic, \\ Shimokato 3046-2, Chuo City, \\ Yamanashi Prefecture 409-3821, \\ Japan \\ ${ }^{2}$ Department of Radiology, \\ University of Yamanashi, Chuo City, \\ Yamanashi Prefecture, Japan \\ Full list of author information is \\ available at the end of the article
}

\begin{abstract}
Background: Using phantoms and clinical studies in prone hanging breast imaging, we assessed the image quality of a commercially available dedicated breast PET $(\mathrm{dbPET})$ at the detector's edge, where mammary glands near the chest wall are located. These are compared to supine PET/CT breast images of the same clinical subjects.
\end{abstract}

Methods: A breast phantom with four spheres (16-, 10-, 7.5-, and 5-mm diameter) was filled with ${ }^{18}$ F-fluorodeoxyglucose solution (sphere-to-background activity concentration ratio, 8:1). The spheres occupied five different positions from the top edge to the centre of the detector and were scanned for $5 \mathrm{~min}$ in each position. Reconstructed images were visually evaluated, and the contrast-to-noise ratio (CNR), contrast recovery coefficient (CRC) for all spheres, and coefficient of variation of the background $\left(\mathrm{CV}_{\mathrm{B}}\right)$ were calculated. Subsequently, clinical images obtained with standard supine PET/CT and prone dbPET were retrospectively analysed. Tumour-tobackground ratios (TBRs) between breast cancer near the chest wall (close to the detector's edge; peripheral group) and at other locations (non-peripheral group) were compared. The TBR of each lesion was compared between dbPET and PET/CT.

Results: Closer to the detector's edge, the CNR and CRC of all spheres decreased while the $C V_{B}$ increased in the phantom study. The disadvantages of this placement were visually confirmed. Regarding clinical images, TBR of dbPET was significantly higher than that of PET/CT in both the peripheral (12.38 \pm 6.41 vs $6.73 \pm 3.5, p=$ $0.0006)$ and non-peripheral (12.44 \pm 5.94 vs $7.71 \pm 7.1, p=0.0183)$ groups. There was no significant difference in TBR of dbPET between the peripheral and non-peripheral groups.

Conclusion: The phantom study revealed poorer image quality at $<2-\mathrm{cm}$ distance from the detector's edge than at other more central parts. In clinical studies, however, the visibility of breast lesions with dbPET was the same regardless of the lesion position, and it was higher than that in PET/CT. dbPET has a great potential for detecting breast lesions near the chest wall if they are at least $2 \mathrm{~cm}$ from the edge of the FOV, even in young women with small breasts.

Keywords: Dedicated breast positron emission tomography, ${ }^{18} \mathrm{~F}$-Fluorodeoxyglucose, Image quality, Edge of detector 


\section{Background}

${ }^{18}$ F-Fluorodeoxyglucose (FDG) positron emission tomography/computed tomography (PET/CT) has become one of the most useful tools in diagnostic imaging for cancer. Many studies have demonstrated the efficacy of whole-body FDG-PET/CT in staging or re-staging, in monitoring the response to therapy, and for predicting the prognosis of patients with breast cancer [1-3]. It is important to detect breast cancer at an early stage when the lesions are small, since mortality increases with tumours exceeding 1 $\mathrm{cm}$ in size $[4,5]$. However, detection of small breast cancers by whole-body PET/CT is challenging because of its limited spatial resolution [6]. High-resolution dedicated breast PET (dbPET) scanners have been developed to detect small breast lesions. There are the two dominant types of high-resolution dbPET, i.e. positron emission mammography (PEM) and a tomographic technique using a ring-shaped scanner (ring-shaped dbPET) [7]. PEM systems depict breast tissue via soft compression of the breast with two opposing plate-like detectors and have higher sensitivity for detecting small lesions than whole-body PET/CT [8-10]. Ring-shaped dbPET scanners do not employ any breast compression and can visualise breast cancer more clearly than whole-body PET/ CT $[11,12]$. These high-resolution breast PET systems have greater photon sensitivity and can improve spatial resolution by setting the detector close to the breast, reducing respiratory movement (either by fixing the breast to the PEM detector or by scanning in the prone position for $\mathrm{dbPET}$ ), and using smaller detection units than those of whole-body PET/CT. Their performances have been evaluated using NEMA-NU4-2008 standards [13], and the physical parameters of dbPET and whole-body PET/CT have been compared using a common breast phantom [14]. In that comparative study, the breast phantom was located at the centre of each scanner, and no studies have reported on the quality of dbPET images close to the edge of the detector. However, many Japanese women have small breasts, and their mammary glands are often located near the chest wall, close to the edge of detector, even when they are in the prone position. This tendency is particularly common in young women who are less likely to have breast ptosis than older women. Therefore, it is necessary to evaluate the consequences of a shift in the position of the breast phantom away from the centre of the detector. This study aimed to confirm the image quality of dbPET at the edge of the detector by phantom and clinical studies and to compare them with clinical PET/CT.

\section{Methods}

This single-institution study was approved by the Institutional Review Board of the Kofu Neurosurgical Hospital and Yamanashi PET imaging clinic in accordance with the Declaration of Helsinki. Because of the retrospective study design and the use of anonymised patient data, the requirement for informed consent was waived.

\section{Ring-shaped dbPET scanner}

The dbPET scanner (Elmammo, Shimadzu Corp., Kyoto, Japan) has received approval from the Japanese Pharmaceutical Affairs Law and is commercially available in Japan. It consists of 36 detector modules arranged in three contiguous rings, has a diameter of $195 \mathrm{~mm}$ and a transaxial length of $156.5 \mathrm{~mm}$, and has depth-of-interaction measurement capability [15]. The transaxial effective field-of-view (FOV) is $185 \mathrm{~mm}$. Each 
detector block consists of a four-layered $32 \times 32$ array of lutetium oxyorthosilicate crystals $(1.44 \mathrm{~mm} \times 1.44 \mathrm{~mm} \times 18 \mathrm{~mm}$ in size with each depth-of-interaction (DOI) layer being $4.5 \mathrm{~mm}$ tall) coupled to a 64-channel position-sensitive photomultiplier tube via a light guide. Attenuation correction was calculated using a uniform attenuation map with object boundaries obtained from emission data [16]. Scatter correction was performed using the convolution-subtraction method with kernels obtained by background tail fitting [17]. Performance metrics included 1.5-mm FWHM resolution in standard mode in the transverse, sagittal, and coronal views, detector sensitivity of $0.09-0.13$ $\mathrm{cps} / \mathrm{Bq}$ at the centre of the detector, and the sensitivity at $39.5 \mathrm{~mm}$ from the edge of the detector (depth of $1 / 4$ ) is $0.05-0.08 \mathrm{cps} / \mathrm{Bq}$. The peak noise equivalent count (NEC) was 600-800 kcps. The sensitivity values and peak NEC are based on the manufacturer's product specifications, which were not measured in this study; therefore, they show slight variations. The characteristics and standard performance of this scanner have been reported in detail previously [13].

\section{Whole-body PET/CT scanner}

PET/CT scans were obtained using a Biograph Horizon TrueV FDG-PET/CT system (Siemens Medical Solutions, Knoxville, TN, USA). This system has 52 detector rings consisting of 160 blocks, with each block containing an array of $13 \times 13$ lutetium oxyorthosilicate crystals $(4 \mathrm{~mm} \times 4 \mathrm{~mm} \times 20 \mathrm{~mm})$ covering an axial FOV of $221 \mathrm{~mm}$ and a transaxial FOV of $690 \mathrm{~mm}$ diameter. A CT scan was performed for attenuation correction $(130 \mathrm{kV} ; 15-70 \mathrm{~mA}$; tube rotation time, $0.6 \mathrm{~s}$ per rotation; pitch, 1 ; a transaxial FOV, $700 \mathrm{~mm}$; and section thickness, $5 \mathrm{~mm}$ ).

\section{Development and preparation of the breast phantom}

A cylindrical breast phantom containing four plastic spheres of different diameters was used. The inner diameter and hight of the cylinder were $100 \mathrm{~mm}$ and $140 \mathrm{~mm}$, respectively. The diameters of the spheres, arranged in a planar circle inside the phantom, were $5,7.5,10$, and $16 \mathrm{~mm}$. Spheres smaller than $5 \mathrm{~mm}$ in diameter were not used because they could not be detected by PET/CT. Furthermore, in our previous studies with low TBR phantoms, the smallest 5-mm-diameter sphere could not be visually detected on dbPET images when the sphere-to-background activity concentration was less than $8: 1$ [14]. Therefore, the visibility of lesions smaller than $5 \mathrm{~mm}$ was not evaluated in this study. The cylinder and four spheres were filled with 18F-FDG solution at a sphere-tobackground radioactivity concentration ratio of $8: 1$ in accordance with a previous study [14]. The background radioactivity at the start of data acquisition by dbPET was set to $2.46 \mathrm{kBq} / \mathrm{mL}$. One scan was performed under each position as detailed in the next section.

\section{Data acquisition and image reconstruction}

The breast phantom was positioned such that the spheres were precisely located in the same transverse plane at different positions in the transverse field of view. The spheres were positioned at $8 \mathrm{~mm}, 13 \mathrm{~mm}, 19.5 \mathrm{~mm}$ (1/8 of detector axial FOV), $39 \mathrm{~mm}(1 / 4$ of detector axial FOV), and $78 \mathrm{~mm}(1 / 2$ or halfway point of the detector axial FOV) below the top edge of the detector (Fig. 1). Since it is unlikely that a breast lesion is located at 


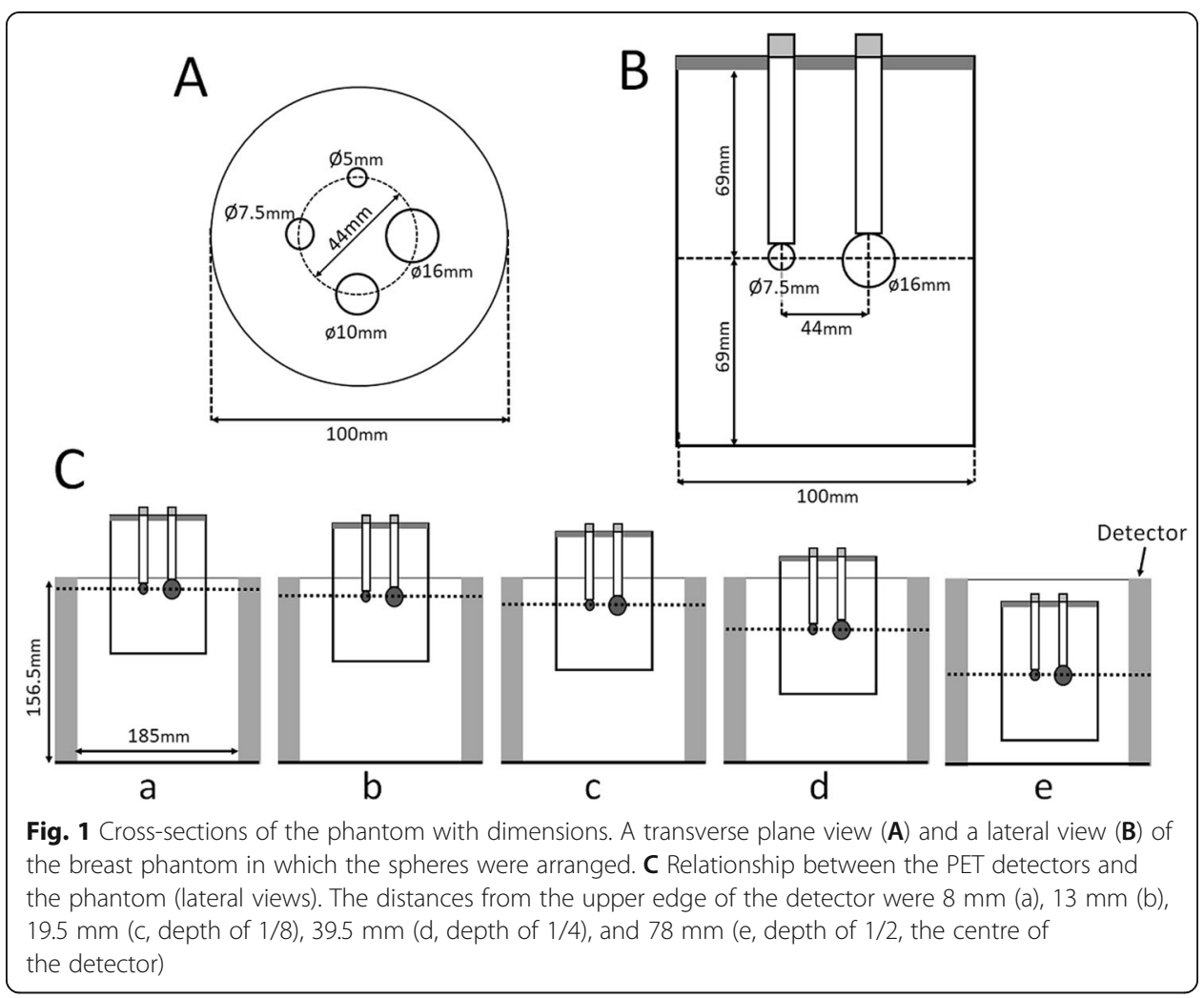

the bottom edge of the detector, only the chest wall side of the detector was evaluated. Sphere placement at each position in the detector was confirmed visually and by measurement on the image. A three-dimensional list-mode dynamic row-action maximumlikelihood algorithm (LM-DRAMA) was applied for the reconstruction of a dbPET image. DRAMA has been shown to achieve fast converge with a reasonable signal-tonoise ratio with a single iteration and 128 subsets by including the relaxation parameter $\lambda$, which was defined by the subset number and the relaxation control parameter of $\beta$ $[18,19]$. In this study, the dbPET images were reconstructed using LM-DRAMA with $\beta$ $=20$, the matrix size in the transverse view $236 \times 200 \times 236$, and a post-reconstruction smoothing Gaussian filter (1.17-mm FWHM) without the time-of-flight (TOF) algorithm according to the previous report [14]. For the clinical images, the extracted contour was the same as the subject's boundary and was therefore used for the attenuation coefficient map without adjustment. For the phantom images, the estimated contour of the boundary was adjusted to account for the wall thickness of the phantom. The reconstructed voxel size of the dbPET images was $0.78 \mathrm{~mm} \times$ $0.78 \mathrm{~mm} \times 0.78 \mathrm{~mm}$.

The clinical PET/CT images were reconstructed using the ordered subset expectation maximisation method and the TOF algorithm with four iterations and 10 subsets. The CT data were resized from a $512 \times 512$ matrix to a $180 \times 180$ matrix to match the PET data and construct CT-based transmission maps for attenuation correction of the PET data with a post-reconstruction smoothing Gaussian filter ( $5 \mathrm{~mm}$ FWHM). The reconstructed voxel size of the PET/CT images was $4.11 \mathrm{~mm} \times 4.11 \mathrm{~mm} \times 5 \mathrm{~mm}$.

The volume per voxel of the dbPET image is $0.47 \mathrm{~mm}^{3}$, while that of the PET/CT image is $84.5 \mathrm{~mm}^{3}$, which is 178 times larger than that of the dbPET. 


\section{Analyses of phantom image quality}

Visual and quantitative analysis of all PET images was performed using an imaging workstation equipped with syngo.via VB10 software (Siemens Healthcare GmbH, Erlangen, Germany). Standardised uptake values (SUVs), as a semi-quantitative assessment of FDG accumulation, were extracted using this software. The SUV of a given tissue was calculated using the following formula:

$$
\mathrm{SUV}=\frac{\text { Tumour activity concentration }(\mathrm{Bq} / \mathrm{ml})}{\text { Injected dose }(\mathrm{Bq})} \times \text { body weight }(\mathrm{g})
$$

The maximum ( $\left.\mathrm{SUV}_{\text {max }}\right)$ and the mean $\left(\mathrm{SUV}_{\text {mean }}\right)$ SUVs are the maximum and average value within the region of interest (ROI) (or volume of interest [VOI]), respectively.

An experienced nuclear medicine physician and two experienced PET technologists evaluated the hot spheres. Evaluations were performed using the slices displayed in the coronal image slice containing the sphere centres. The 5-mm-diameter hot sphere was visually graded as follows: 2, identifiable; 1, visualised, but similar hot spots observed elsewhere; and 0 , not visualised. Spheres with visual scores of $\geq 1.5$ were deemed to be detectable. The final score for the visibility of the smallest sphere was the mean of the scores from three readers. The visual assessment was performed based on the Japanese guidelines [20]. A circular ROI with a diameter of $5 \mathrm{~mm}$ was placed on the central slice of the 5-mm hot sphere. Additionally, 12 ROIs with a diameter of $5 \mathrm{~mm}$ were placed in the background region of the coronal image slice that contained the sphere centres, and 12 ROIs were placed in the +5 -mm and - 5-mm adjacent slices (36 ROIs in total). The contrast-to-noise ratio (CNR) and contrast recovery coefficient (CRC) were calculated to quantitatively compare the visibility between the different positions in the dbPET detector. CNR and CRC provide information about the visibility and how accurately the system reproduces the true activity concentration, respectively. A modified CNR was calculated as follows:

$$
\mathrm{CNR}=\frac{\left|C_{\mathrm{H}}-\overline{C_{\mathrm{B}, 5 \mathrm{~mm}}}\right|}{\mathrm{SD}_{\mathrm{B}, 5 \mathrm{~mm}}}
$$

where $C_{\mathrm{H}}$ is the $\mathrm{SUV}_{\text {mean }}$ in each sphere ROI, $\overline{C_{\mathrm{B}, 5 \mathrm{~mm}}}$ is the average $\mathrm{SUV}_{\text {mean }}$ of the background ROIs, and $\mathrm{SD}_{\mathrm{B}}, 5 \mathrm{~mm}$ is the standard deviation of the background ROIs.

A modified CRC was calculated as follows:

$$
\mathrm{CRC}=\frac{\left(C_{\mathrm{H}} / \frac{1}{C_{\mathrm{B}, 5 \mathrm{~mm}}}\right)-1}{\left(a_{\mathrm{H}} / a_{\mathrm{B}}\right)-1} \times 100[\%],
$$

where $a_{\mathrm{H}}$ and $a_{\mathrm{B}}$ are the activity concentration in the hot sphere and the background, respectively.

We also placed 10 ROIs with a diameter of $16 \mathrm{~mm}$ in the background region of the coronal image slice that contained the sphere centres and its $+5-\mathrm{mm}$ and $-5-\mathrm{mm}$ adjacent slices (30 ROIs in total).

The modified coefficient of variation $\left(\mathrm{CV}_{\mathrm{B}}\right)$ was calculated using the data from these $16 \mathrm{~mm}$ ROIs as follows:

$$
\mathrm{CVB}=\frac{\mathrm{SD}_{\mathrm{B}, 16 \mathrm{~mm}}}{\bar{C}_{\mathrm{B}, 16 \mathrm{~mm}}} \times 100[\%]
$$

where $\mathrm{SD}_{\mathrm{B}}, 16 \mathrm{~mm}$ is the standard deviation in the background ROIs and $\overline{C_{\mathrm{B}, 16 \mathrm{~mm}}}$ is the average $\mathrm{SUV}_{\text {mean }}$ of the background ROIs. 
These physical values were calculated according to previous reports [14, 21].

\section{Analysis of human images}

Of a total of 202 consecutive women who underwent both dbPET and whole-body PET/CT scans from August 2016 to September 2019, 62 histologically proven breast cancer tumours of 57 women with positive findings on both dbPET and whole-body $\mathrm{PET} / \mathrm{CT}$ images were included in the study. Patients fasted at least $6 \mathrm{~h}$ prior to administration of $18 \mathrm{~F}-\mathrm{FDG}(3 \mathrm{MBq} / \mathrm{kg}$ ) and were scanned at $60 \mathrm{~min}$ post-injection by wholebody PET/CT in the supine position for $90 \mathrm{~s}$ per bed position. Then, they were scanned at $90 \mathrm{~min}$ post-injection by $\mathrm{dbPET}$ in the prone position for $7 \mathrm{~min}$ per breast. The PET/CT and dbPET images were reconstructed using the same conditions as for the phantom images.

All PET images were evaluated separately by two experienced nuclear medicine physicians (with 16 and 7 years of experience in interpreting PET, respectively). Of the 62 lesions, those in which the shortest distance from the detector edge on the chest wall side to the tumour centre was $2 \mathrm{~cm}$ or less on the transverse image of dbPET were defined as the "peripheral group"; the other lesions were defined as the "non-peripheral group". Non-mass uptakes, other than focus and mass-like uptakes, were excluded because their quantitative reliability could not be established. Tumours that were exactly centred in both peripheral and non-peripheral regions and whose volume was equally present in both regions were also excluded.

The quantitative value of PET is known to be affected by the partial volume effect [22]. To account for lesion size bias, lesion sizes were matched in the peripheral and non-peripheral groups. The non-peripheral group was reorganised such that lesion size matched the peripheral group in a one-to-one correspondence. As a result, 23 lesions in each group (total 46 lesions) were included in the final analysis.

To evaluate lesion visibility in dbPET depending on the position of the tumour, tumour-to-background ratio (TBR) was calculated as follows. All PET images were displayed in an inverse grey scale with a standardised uptake range of $0-6$ for the purpose of reducing intra-reader variability. First, the smallest spheroid VOI that just contained the tumour was placed on the monitor. Second, 5-mm-diameter spherical VOIs were placed at 6 locations on the top, bottom, left, right, anterior, and posterior of the tumour, as close as possible to it, in the non-peripheral group. Five VOIs were used in the peripheral group; the posterior VOI was excluded because there was not enough space to place it posterior of the tumour (Fig. 2). The TBR was the $\mathrm{SUV}_{\max }$ of the VOI on the tumour divided by the average $\mathrm{SUV}_{\text {mean }}$ of the five or six VOIs on the background.

$$
\mathrm{TBR}=\frac{\mathrm{SUV}_{\text {max } \_\mathrm{T}}}{\mathrm{SUV}_{\text {mean } \_\mathrm{B}}}
$$

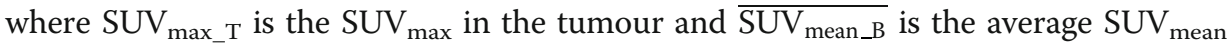
of the background VOIs.

In PET/CT, the $\mathrm{SUV}_{\text {max }}$ and the $\mathrm{SUV}_{\text {mean }}$ of the same ROI are equal because a 5mm-diameter spherical VOI contains only one voxel. The TBRs were compared between $\mathrm{dbPET}$ and PET/CT images, and the TBR of dbPET was compared between the peripheral and non-peripheral groups. 


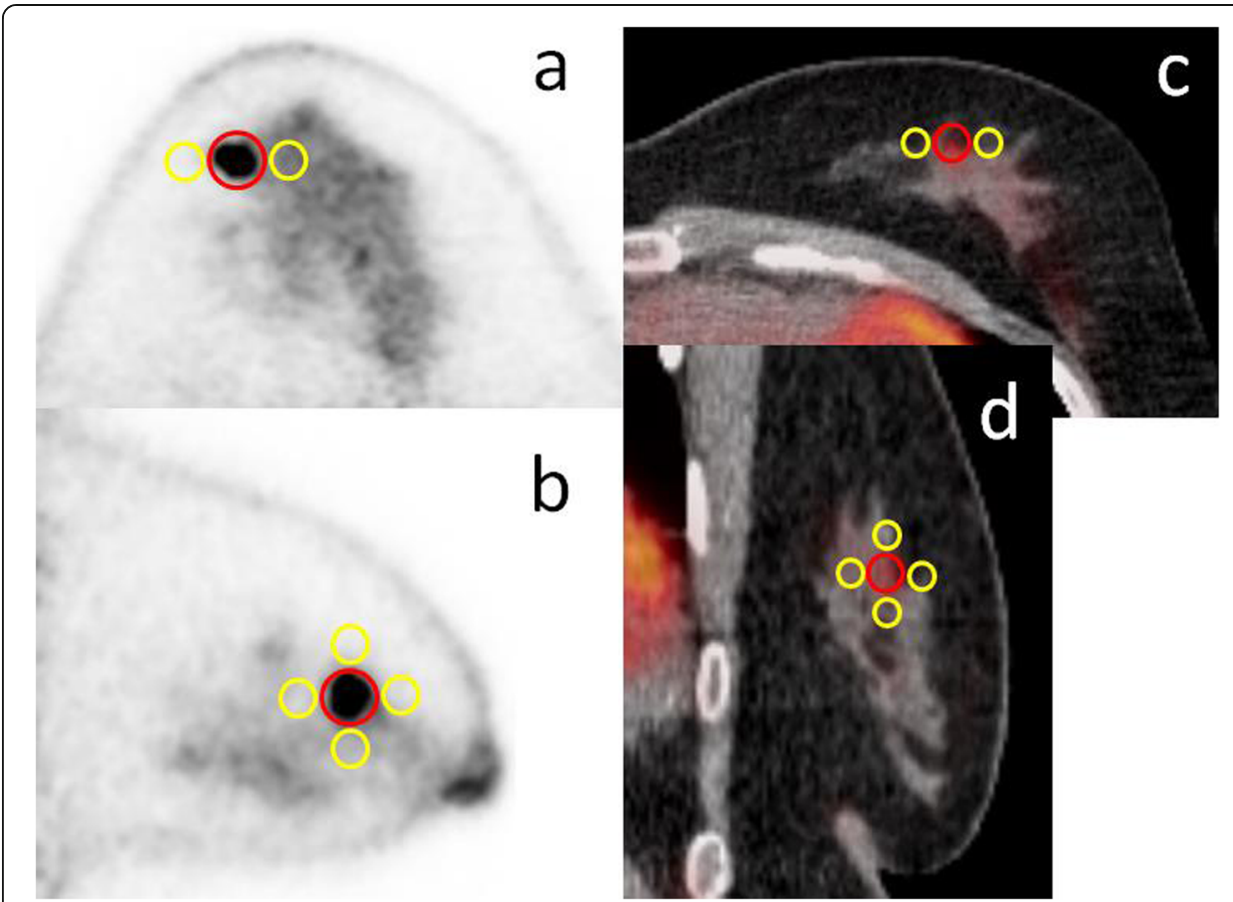

Fig. 2 Positioning of the volume of interest (VOI) for the measurement of clinical PET images. A spherical VOI (red) on FDG uptake of the tumour and 5 (or 6 ) spherical VOls (yellow) were placed to calculate the TBR. Transaxial and sagittal images of $\operatorname{dbPET}(\mathbf{a}, \mathbf{b})$ and whole-body PET/CT $(\mathbf{c}, \mathbf{d})$

\section{Statistical analysis}

A paired $t$ test was used to compare the TBR of dbPET and whole-body PET/CT for the peripheral and non-peripheral groups, respectively. The Mann-Whitney $U$ test was used to test for differences in TBR on dbPET between peripheral and non-peripheral lesion groups. Statistical significance was defined as $p<0.05$. Additionally, for these PET measurements, interclass correlation coefficients (ICC) were used to evaluate the reliability between readers.

\section{Results}

\section{dbPET phantom studies}

Images of the breast phantom scanned by dbPET at the five different positions are shown in Fig. 3. In the qualitative evaluation, the visual scores recorded by a nuclear medicine physician and two nuclear medicine technologists on the dbPET images at 8 $\mathrm{mm}, 13 \mathrm{~mm}, 19.5 \mathrm{~mm}$ (depth of 1/8), $39 \mathrm{~mm}$ (depth of 1/4), and $78 \mathrm{~mm}$ (depth of $1 / 2$, the centre of the detector) below the top edge of the detector were $0,0.33,1.67,2$, and 2 , respectively. All other spheres had visual scores of 2 . Second, in the quantitative evaluations, the $\mathrm{CNR}, \mathrm{CRC}$, and $\mathrm{CV}_{\mathrm{B}}$ for the 5-mm sphere at the centre of the detector were 10.96, 10.02, and 5.91, respectively (Fig. 4). The CNR and CRC decreased and the $\mathrm{CV}_{\mathrm{B}}$ increased when the phantom was placed closer to the detector's edge. Image degradation closer to the edge of the detector was confirmed by visual scoring. Based on the results of this phantom study, the boundary line between peripheral and nonperipheral lesions in clinical studies was defined as $2 \mathrm{~cm}$ from the upper edge of the detector. 


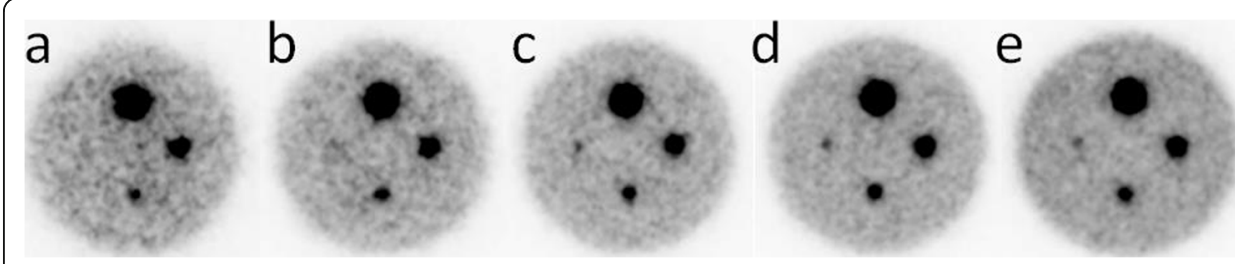

Fig. 3 Images of the breast phantom scanned by dbPET at the five different positions. Images $\mathbf{a}, \mathbf{b}, \mathbf{c}, \mathbf{d}$, and e correspond to phantom images scanned at $8 \mathrm{~mm}, 13 \mathrm{~mm}, 19.5 \mathrm{~mm}$ (depth of 1/8), $39 \mathrm{~mm}$ (depth of 1/4), and $78 \mathrm{~mm}$ (depth of $1 / 2$, the centre of the detector) below the top edge of the detector. Closer to the edge of the detector, the background was noisier. The hot spots of the smallest sphere with a diameter of $5 \mathrm{~mm}$ could not be confirmed in $\mathbf{a}$ and $\mathbf{b}$

\section{Patient studies}

A total of 46 lesions (23 in each group) in 45 breasts of 44 patients (age range 37-87 years, mean 57.8 years) were evaluated. One patient had one peripheral and one nonperipheral lesion on one side of her breast, one patient had two peripheral lesions on one side of the breast, and each of the 42 patients had one lesion.

After propensity score matching for lesion size, the mean diameters of the lesions in the peripheral and non-peripheral groups were $19.3 \pm 12 \mathrm{~mm}$ and $20 \pm 12.2 \mathrm{~mm} \mathrm{(p=}$ 0.7663), respectively (Table 1$)$. The ICC of the TBR was excellent (0.92 for PET/CT and 0.89 for $\mathrm{dbPET}$ ). The average values evaluated by two readers were analysed in this study. The TBR of dbPET was significantly higher than that of whole-body PET/CT in both the peripheral $(12.38 \pm 6.41$ vs $6.73 \pm 3.5, p=0.0006)$ and non-peripheral groups $(12.44 \pm 5.94$ vs $7.71 \pm 7.1, p=0.0183)$ (Fig. 5a). There was no significant difference in the TBRs of dbPET between the peripheral and non-peripheral ( $p=0.8261$, Fig. $5 \mathrm{~b})$. Figure 6 shows representative cases of peripheral and non-peripheral breast cancer acquired by dbPET in the prone position and PET/CT in the supine position. The breast cancers were visualised on dbPET more easily than on PET/CT regardless of the location of the lesion (peripheral or non-peripheral).

\section{Discussion}

In this study, we evaluated the image quality obtained at different coronal locations within the dbPET detector for ring-type breast PET. In the phantom study, the CNRs

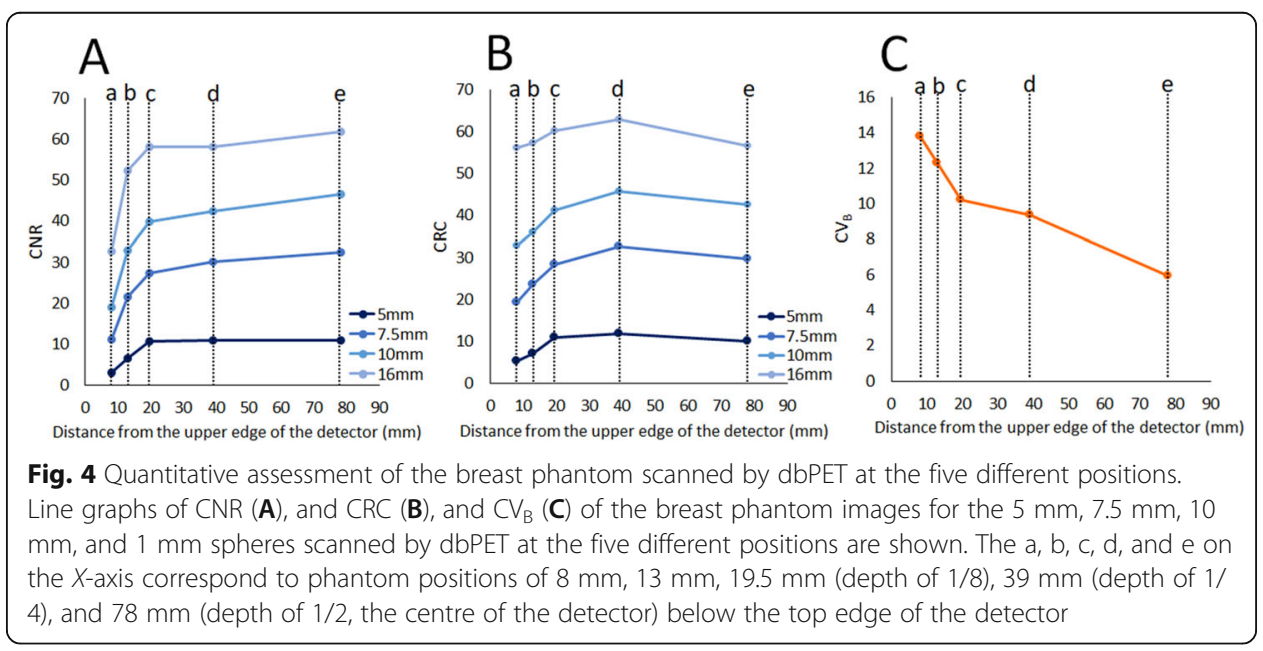


Table 1 Characteristics of the 46 lesions in 44 patients

\begin{tabular}{|c|c|c|c|}
\hline \multicolumn{2}{|l|}{ Group } & Peripheral & Non-periphera \\
\hline \multicolumn{2}{|c|}{ Number of lesions (women) } & $23(22)$ & $23(23)$ \\
\hline \multicolumn{2}{|c|}{ Age (years) [median, (range)] } & $52(37-87)$ & $62(43-79)$ \\
\hline \multicolumn{2}{|c|}{ Clinical size (mm) [median, (range)] } & $17(7-51)$ & $17(7-52)$ \\
\hline \multicolumn{2}{|c|}{ Distance from chest wall to lesion $(\mathrm{mm})[\text { median, (range) }]^{\mathrm{a}}$} & $0.83(0.44-1.55)$ & $32.7(20.2-64.7)$ \\
\hline \multirow[t]{5}{*}{ Histopathology } & Invasive ductal carcinoma & 19 & 20 \\
\hline & Invasive lobular carcinoma & 1 & 0 \\
\hline & Invasive ductal and lobular carcinoma & 1 & 0 \\
\hline & Other invasive carcinomas & 1 & 0 \\
\hline & Ductal carcinoma in situ & 1 & 3 \\
\hline \multirow[t]{4}{*}{ Subtype } & Luminal A/B & $9 / 7$ & $10 / 5$ \\
\hline & HER2 positive & 1 & 2 \\
\hline & Triple negative & 2 & 3 \\
\hline & Unknown & 4 & 3 \\
\hline
\end{tabular}

${ }^{\mathrm{a}}$ Distance from the FOV margin on the chest wall to the centre of the lesion

and CRCs were lower and the $\mathrm{CV}_{\mathrm{Bs}}$ were higher for all sphere sizes closer to the breast-insert side of the detector. These results indicate that the quantitative image quality was degraded at about $2 \mathrm{~cm}$ from the breast-insert or chest wall side of the detector.

Minoura et al. reported that dbPET images show high levels of noise at the edge of the detector (the top of the detector or the chest wall side) and showed the relationship between the slice position in the dbPET image and the standard deviation of noise [23]. Our results showing that dbPET image quality decreases at $19.5 \mathrm{~mm}$ from the detector edge are consistent with their reports. The geometric efficiency by Monte Carlo simulation at this depth was 0.2 , which was considerably lower than that at the centre, which was 0.65. Usually, whole-body PET scans use overlapping acquisition beds to correct for reduced sensitivity at the detector edges; acquisition of data in overlapped regions

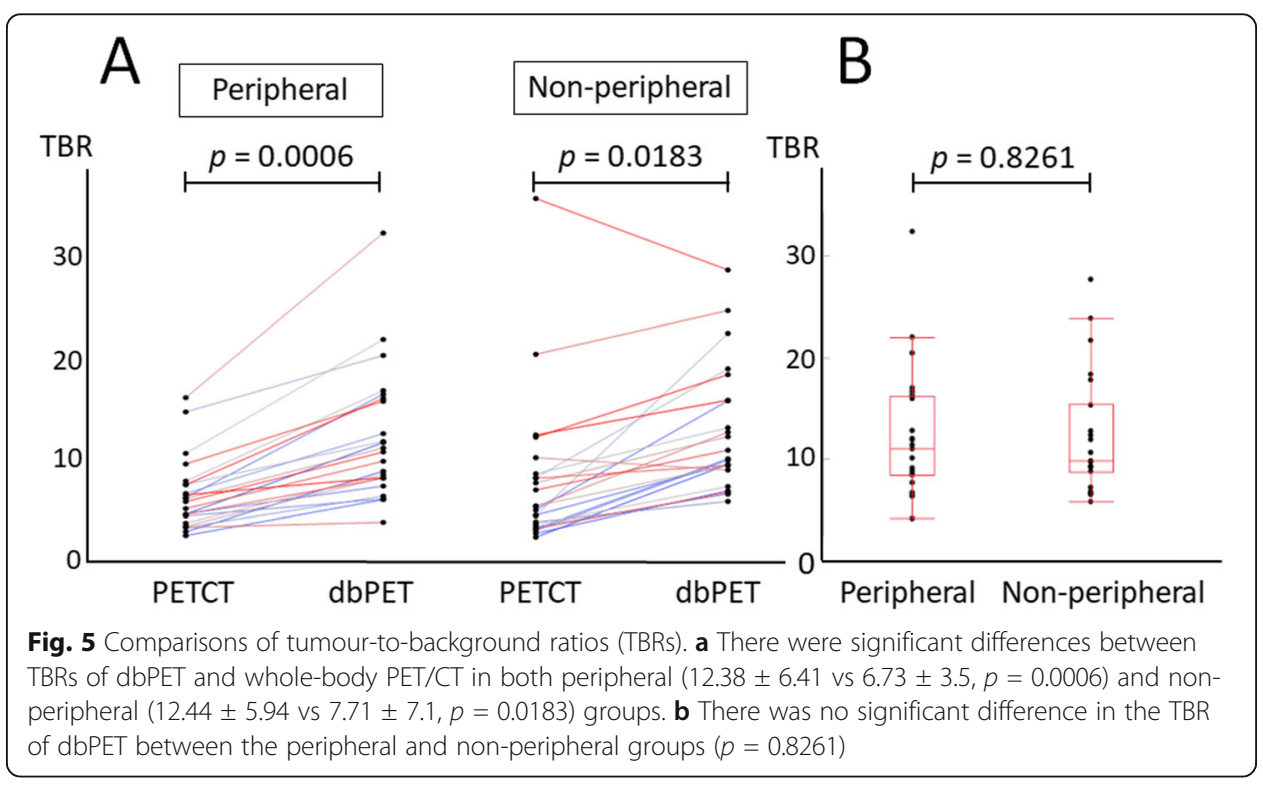




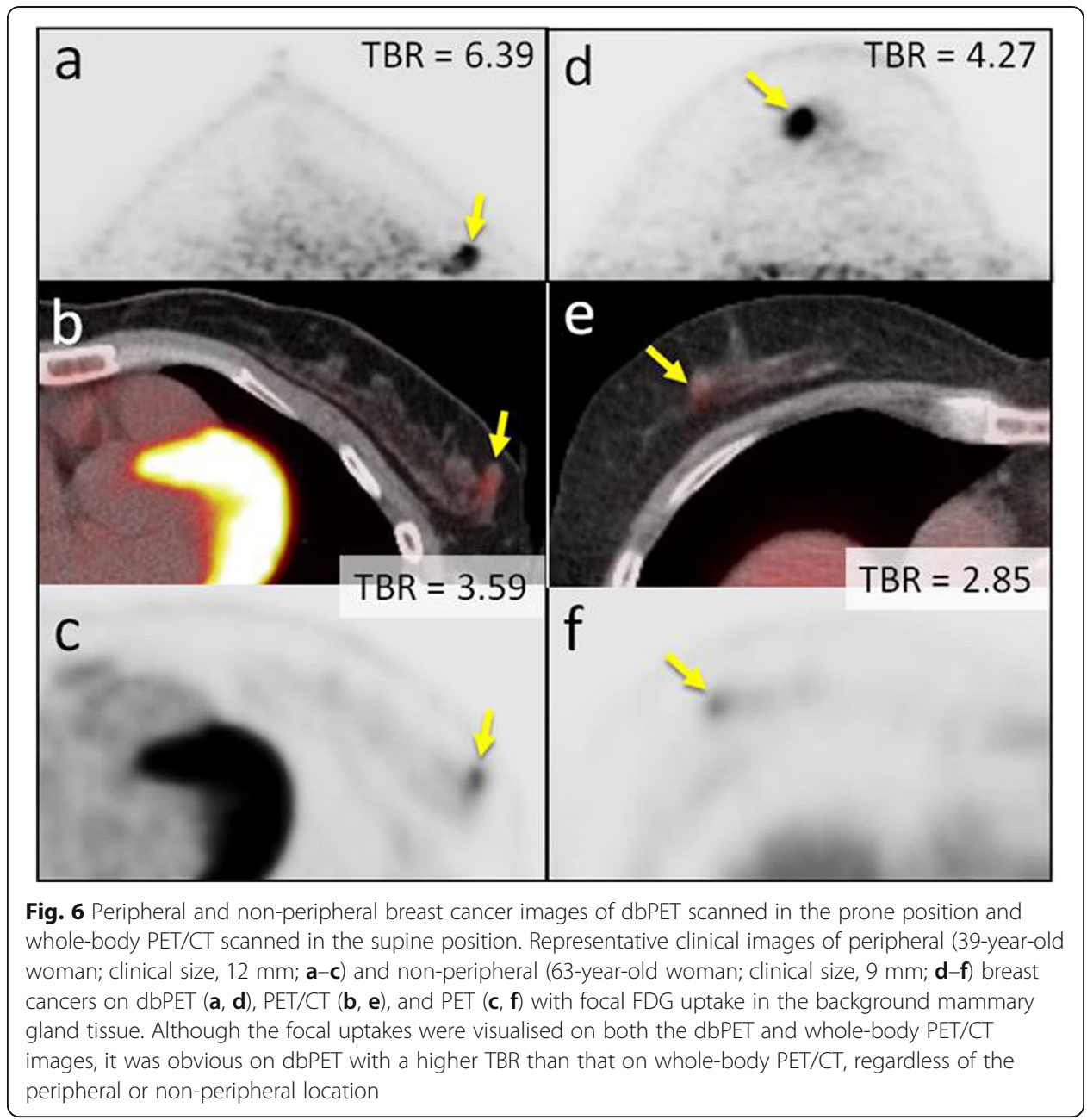

can improve quantitative accuracy $[24,25]$. However, since the dbPET scanner is fixed and cannot use overlapping acquisition to improve image quality near the edges of the detector, there are concerns that important information may be missed. Additionally, out-of-FOV radioactivity, among which myocardial uptake may be most significant, would also significantly affect image quality. However, the effect of out-of-FOV radioactivity on the dbPET image quality could not be evaluated in this phantom study because such structures that showed high FDG uptake, such as the myocardium, were not included in our phantom. Therefore, to better reproduce the same scatter, singles, and random conditions as in real patients, evaluation with a phantom that simulates out-of-FOV radioactivity from the patients' chest is necessary, which may detrimentally affect these results.

Based on the phantom test results, the lesion visibility of clinical dbPET images was compared for peripheral lesions located up to $2 \mathrm{~cm}$ from the upper edges of the detector and for the other non-peripheral lesions, which revealed that CNR and CRC decreased and $\mathrm{CV}_{\mathrm{B}}$ increased as the lesions were positioned closer to the $2 \mathrm{~cm}$ edge of the dbPET scanner FOV. While in the clinical study, there were significant differences between PET/CT and dbPET TBRs in both groups. There were no significant differences between the peripheral and non-peripheral groups for dbPET. Peripheral and 
non-peripheral groups could not be compared in whole-body PET/CT, since the patients were scanned in the supine position, with their breasts naturally slumped against their chest walls. The clinical dbPET images had a high TBR in some projection directions, which may have facilitated the detection of lesions. This may be because the phantom image had a uniform background, whereas human breasts have different proportions of mammary glands and fat, and therefore, the physiological FDG uptake in the background tissue was not uniform [26, 27]. Additionally, the TBRs in both dbPET groups were significantly higher than that in PET/CT. dbPET is a higher-resolution scanner than conventional whole-body PET/CT, and the prone position significantly supresses respiratory movements compared to whole-body PET/CT scans; therefore, even if the lesion is located at the edge of the detector, dbPET may show higher lesion visibility than $\mathrm{PET} / \mathrm{CT}$.

dbPET achieves higher geometric sensitivity and spatial resolution than whole-body PET/CT by (i) DOI detector and smaller voxel size in the former than in the latter, (ii) reduction of respiratory movement of the breast by acquisition in the prone position, and (iii) bringing the detector close to the breast. Especially, the 4-layer DOI detector used in dbPET can maintain geometric sensitivity and spatial resolution at the edges of the coronal field of view [28, 29]. The 4-layer DOI detector can detect gamma-ray interaction positions accurately by detecting Compton scattering events, and DOI information can dramatically reduce the voxel size. The voxel volume of dbPET $(0.47$ $\mathrm{mm}^{3}$ ) image was 178 times lower than that of PET/CT $\left(84.5 \mathrm{~mm}^{3}\right)$ image in this study. Additionally, when the background mammary gland showed physiological FDG uptake, the measured dbPET contrast was higher than the measured PET/CT contrast in the same lesion. As a result of earlier studies [30-32], the 2018 edition of the Japanese Guidelines for the Practice of Breast Cancer newly described the use of high-resolution breast PET as a supplemental modality for breasts with high density on mammography. Consequently, dbPET is expected to be applied to young women who often have highdensity breasts. Both dbPET and PEM have the disadvantage that, due to their structural features, a part of the mammary gland near the chest wall is in a blind area and the lesion may be outside the field of view. However, this study demonstrates that if the lesion is within the field of view of dbPET, it can be detected with high probability, beyond $2 \mathrm{~cm}$ from the edge of the detector. Further studies are needed to classify in which patients and/or what lesions are likely to be located outside the FOV of either dbPET or PEM.

Because dbPET has much better performance characteristics, the lesions that can be detected by PET/CT would be more easily visualised. However, given the prognosis of breast cancer, comparison between both systems should focus on smaller lesions. The spatial resolution of whole-body PET/CT has improved due to the development of reconstruction techniques such as TOF and point spread function (PSF) modelling algorithms. In this study, we quantitatively evaluated TOF-reconstructed PET/CT images, since edge artefacts are known to occur in PSF modelling during reconstruction and are significant for small lesions [33]. Furthermore, some reports have shown that visualisation of small breast lesions can be improved by performing PET/CT in the prone position using assistive devices to allow breast expansion and suppression of respiratory movements [34, 35]. PET technologies, such as TOF and PSF, smaller pixel sizes, and prone position scanning, are expected to improve the visual detection rate of smaller 
lesions using PET/CT. This will allow a more direct future comparison of both devices for smaller lesions.

Our study had several limitations. First, the phantom was scanned only once for each position. The reproducibility of the findings would have been better if the average results of several scans under each condition were calculated. Second, the clinical study design was retrospective, and the patient cohort was small. Because only histologically proven breast cancers were included in this clinical study, small breast cancers near the edge of the detector that are false-negative on PET may not be sufficiently evaluated. Studies with larger populations and considerations including histology and subtypes of breast cancer will be required to address these limitations. Third, PET images acquired 90 min after injection are known to have improved uptake and contrast compared to those acquired $60 \mathrm{~min}$ after injection. Because this study was a retrospective study, all patients were scanned 60 and $90 \mathrm{~min}$ after FDG intravenous injection with PET/CT and $\mathrm{dbPET}$, respectively, under our clinical conditions. This would likely have caused some bias in the results. Randomised prospective studies that appropriately control the start time of the scan are necessary for an accurate comparison of both devices.

\section{Conclusion}

In our phantom study, image quality for all lesion sizes was worse when the phantom was within $2 \mathrm{~cm}$ of the edge of the detector. In the clinical studies, however, lesion visibility was the same regardless of whether the lesion was peripheral or non-peripheral, and the lesion visibility in both conditions was statistically significantly higher for dbPET than that in PET/CT.

\section{Abbreviations}

CT: Computed tomography; FOV: Field of view; ICC: Interclass correlation coefficients; PEM: Positron emission mammography; PET: Positron emission tomography/computed tomography; PSF: Point spread function; ROI: Region of interest; TBR: Tumour-to-background ratio; TOF: Time-of-flight; VOI: Volume of interest

\section{Acknowledgements}

The authors gratefully acknowledge the assistance of Takao Arai from Yamanashi PET Imaging Clinic in the acquisition of the images.

Authors' contributions

All authors contributed to the study design. YS and MI contributed to data collection and analysis. YS wrote the manuscript, and the other authors revised the manuscript. All authors read and approved the final manuscript.

Funding

This research did not receive any specific grant from funding agencies in the public or not-for-profit sectors.

Availability of data and materials

The datasets used and/or analysed during the current study are available from the corresponding author on reasonable request.

Ethics approval and consent to participate

All procedures performed in studies involving human participants were in accordance with the ethical standards of the Institutional Review Board of the Kofu Neurosurgical Hospital and Yamanashi PET imaging clinic (approved on 7 March 2020) and with the 1964 Helsinki Declaration and its later amendments or comparable ethical standards. The requirement for informed consent from each patient has been waived due to its retrospective nature.

Consent for publication

Not applicable. 


\section{Author details}

${ }^{1}$ Yamanashi PET Imaging Clinic, Shimokato 3046-2, Chuo City, Yamanashi Prefecture 409-3821, Japan. ${ }^{2}$ Department of Radiology, University of Yamanashi, Chuo City, Yamanashi Prefecture, Japan. ${ }^{3}$ Department of Radiology, Kofu-kyoritsu Hospital, Kofu City, Yamanashi Prefecture, Japan.

Received: 6 April 2020 Accepted: 22 December 2020

Published online: 18 January 2021

\section{References}

1. Groheux D, Espié M, Giacchetti S, Hindié E. Performance of FDG PET/CT in the clinical management of breast cancer. Radiology. 2013;266:388-5.

2. Lee H, Lee DE, Park S, Kim TS, Jung SY, Lee S, et al. Predicting response to neoadjuvant chemotherapy in patients with breast cancer: combined statistical modeling using clinicopathological factors and FDG PET/CT texture parameters. Clin Nucl Med. 2019;44:21-9.

3. Satoh Y, Nambu A, Ichikawa T, Onishi H. Whole-body total lesion glycolysis measured on fluorodeoxyglucose positron emission tomography/computed tomography as a prognostic variable in metastatic breast cancer. BMC Cancer. 2014; $14: 525$.

4. Ichizawa N, Fukutomi T, Iwamoto E, Akashi-Tanaka S. Long-term results of T1a, T1b and T1c invasive breast carcinomas in Japanese women: validation of the UICC T1 subgroup classification. Jpn J Clin Oncol. 2002;32:108-9.

5. Saadatmand S, Bretveld R, Siesling S, Tilanus-Linthorst MM. Influence of tumour stage at breast cancer detection on survival in modern times: population based study in 173,797 patients. BMJ. 2015;351:h4901.

6. Kumar R, Chauhan A, Zhuang H, Chandra P, Schnall M, Alavi A. Clinicopathologic factors associated with false negative FDG-PET in primary breast cancer. Breast Cancer Res Treat. 2006;98:267-74.

7. Bowen SL, Wu Y, Chaudhari AJ, Fu L, Packard NJ, Burkett GW, et al. Initial characterisation of a dedicated breast PET/CT scanner during human imaging. J Nucl Med. 2009;50:1401-8.

8. Kalinyak JE, Berg WA, Schilling K, Madsen KS, Narayanan D, Tartar M. Breast cancer detection using high-resolution breast PET compared to whole-body PET or PET/CT. Eur J Nucl Med Mol Imaging. 2014:41:260-75.

9. Yamamoto Y, Ozawa Y, Kubouchi K, Nakamura S, Nakajima Y, Inoue T. Comparative analysis of imaging sensitivity of positron emission mammography and whole-body PET in relation to tumor size. Clin Nucl Med. 2015:40:21-5.

10. Fowler AM. A molecular approach to breast imaging. J Nucl Med. 2014;55:177-80.

11. Nishimatsu K, Nakamoto Y, Miyake KK. Higher breast cancer conspicuity on dbPET compared to WB-PET/CT. Eur J Radiol. 2017;90:138-45.

12. Nakamoto R, Nakamoto $Y$, Ishimori T. Diagnostic performance of a novel dedicated breast PET scanner with C-shaped ring detectors. Nucl Med Commun. 2017;38:388-95.

13. Miyake KK, Matsumoto K, Inoue M. Performance evaluation of a new dedicated breast PET scanner using NEMA NU42008 Standards. J Nucl Med. 2014:55:1198-203.

14. Satoh Y, Motosugi U, Imai M, Onishi H. Comparison of dedicated breast positron emission tomography and whole-body positron emission tomography/computed tomography images: a common phantom study. Ann Nucl Med. 2019;34:1-9.

15. Qi J, Kuo C, Huesman RH, Klein GJ, Moses WW, Reutter BW. Comparison of rectangular and dual-planar positron emission mammography scanners. IEEE Trans Nucl Sci. 2002;49:2089-96.

16. Yamakawa Y, Kitamura K. Attenuation correction using level set method for application specific PET scanners. IEEE Nucl Sci Symp Conf Rec. 2011;3130-2. https://doi.org/10.1109/NSSMIC.2011.6152569.

17. Bailey DL, Meikle SR. A convolution-subtraction scatter correction method for 3D PET. Phys Med Biol. 1994;39:411-24

18. Tanaka E, Kudo H. Optimal relaxation parameters of DRAMA (dynamic RAMLA) aiming at one-pass image reconstruction for 3D-PET. Phys Med Biol. 2010;55:2917-39.

19. Tanaka $\mathrm{E}$, Kudo H. Subset-dependent relaxation in block-iterative algorithms for image reconstruction in emission tomography. Phys Med Biol. 2003;48:1405-22.

20. Hosono M, Saga T, Ito K. Clinical practice guideline for dedicated breast PET. Ann Nucl Med. 2014;28:597-602.

21. Øen SK, Aasheim LB, Eikenes L, Karlberg AM. Image quality and detectability in Siemens Biograph PET/MRI and PET/CT systems-a phantom study. EJNMMI Phys. 2019;6:16.

22. Srinivas SM, Dhurairaj T, Basu S, Bural G, Surti S, Alavi A. A recovery coefficient method for partial volume correction of PET images. Ann Nucl Med. 2009;23:341-8.

23. Minoura N, Teramoto A, Ito A, Yamamuro O, Nishio M, Saito K, et al. A complementary scheme for automated detection of high-uptake regions on dedicated breast PET and whole-body PET/CT. Radiol Phys Technol. 2019;12:260-7.

24. Fahey FH. Data acquisition in PET imaging. J Nucl Med Technol. 2002:30:39-49.

25. Panin VY, Smith AM, Hu J, Kehren F, Casey ME. Continuous bed motion on clinical scanner: design, data correction, and reconstruction. Phys Med Biol. 2014:59:6153-74.

26. Vranjesevic D, Schiepers C, Silverman DH, Quon A, Villalpando J, Dahlbom M, et al. Relationship between 18 F-FDG uptake and breast density in women with normal breast tissue. J Nucl Med. 2003;44:1238-42.

27. Mann SD, Perez KL, McCracken EK, Shah JP, Wong TZ, Tornai MP. Initial in vivo quantification of Tc-99m sestamibi uptake as a function of tissue type in healthy breasts using dedicated breast SPECT-CT. J Oncol. 2012:146943.

28. Yamaya T, Yoshida E, Obi T, Ito H, Yoshikawa K, Murayama H. First human brain imaging by the jPET-D4 prototype with a pre-computed system matrix. IEEE Trans Nucl Sci. 2008;55:2482-92.

29. Akamatsu G, Tashima H, Iwao Y, Wakizaka H, Maeda T, Mohammadi A, et al. Performance evaluation of a whole-body prototype PET scanner with four-layer DOI detectors. Phys Med Biol. 2019;64:095014.

30. Caldarella R, Treglia G, Giordano A. Diagnostic performance of dedicated breast positron emission mammography using fluorine-18-fluolodeoxyglucose in women with suspicious breast lesions: a meta-analysis. Clin Breast Cancer. 2014;14 241-8.

31. Yamamoto Y, Tasaki Y, Kuwada Y, Ozawa Y, Inoue T. A preliminary report of breast cancer screening by poitron emission mammography. Ann Nucl Med. 2016;30:130-7. 
32. Koo HR, Moon WK, Chun IK, Eso JA, Jeyanth JX, Chang JM, et al. Background 18F-FDG uptake in positron emission mammography (PEM): correlation with mammographic density and background parenchymal enhancement in breast MRI. Eur J Radiol. 2013;82:1738-42.

33. Kidera D, Kihara K, Akamatsu G, Mikasa S, Taniguchi T, Tsutsui Y, et al. The edge artifact in the point-spread functionbased PET reconstruction at different sphere-to-background ratios of radioactivity. Ann Nucl Med. 2016;30:97-103.

34. Williams JM, Rani SD, Li X, Arlinghaus LR, Lee TC, LR MD, et al. Comparison of prone versus supine 18F-FDG-PET of locally advanced breast cancer: phantom and preliminary clinical studies. Med Phys. 2015;42:3801-13.

35. Teixeira SC, Koolen BB, Vogel WV, Wesseling J, Stokkel MP, Vrancken Peeters MJ, et al. Additional prone 18F-FDG PET/CT acquisition to improve the visualization of the primary tumor and regional lymph node metastases in stage II/III breast cancer. Clin Nucl Med. 2016;41:e181-6.

\section{Publisher's Note}

Springer Nature remains neutral with regard to jurisdictional claims in published maps and institutional affiliations.

Submit your manuscript to a SpringerOpen ${ }^{0}$ journal and benefit from:

- Convenient online submission

- Rigorous peer review

- Open access: articles freely available online

High visibility within the field

- Retaining the copyright to your article

Submit your next manuscript at $>$ springeropen.com 